\title{
INVARIANCE OF PROJECTIONS IN THE DIAGONAL OF A NEST ALGEBRA
}

\author{
JOHN DAUGHTRY
}

(Communicated by Paul S. Muhly)

\begin{abstract}
The study of operator factorization along commutative subspace lattices which are not nests leads to the investigation of the mapping $\phi_{A}$ which takes an orthogonal projection $Q$ in the diagonal of a nest algebra $A$ to the projection on the closure of the range of $A Q$ for certain bounded linear operators $A$. The purpose of this paper is to demonstrate that if $B$ is an operator leaving the range of $Q$ invariant, $V$ is an element of the "Larson radical" of $A, B+V$ is invertible, $(B+V)^{-1}$ belongs to $A$, and $\phi_{B+V}(Q)$ is in the diagonal of $A$, then $\phi_{V}(Q) \leq Q$. For example, if $V$ is in the Jacobson radical of $A$ and $\lambda$ is a nonzero scalar, it follows that $\phi_{\lambda I+V}(Q)=Q$ if and only if $\phi_{\lambda I+V}(Q)$ belongs to the diagonal of $A$. Examples of the applications to operator factorization and unitary equivalence of sets of projections are given.
\end{abstract}

Theorem 1 of [2] gives a fact about the projections in the diagonal of a nest algebra which is closely related to the main result of this paper. The theorem in [2] has significant consequences for the theory of nonanticipative representations of Gaussian random fields, but its applicability to more general problems of operator factorization relative to a commutative subspace lattice is limited by a technical hypothesis relating the nest whose diagonal projections are to be studied to an operator $A$. The purpose of this investigation is to substitute for that technical hypothesis the more natural condition that $A=\lambda I+V$ for some scalar $\lambda$ and some $V$ in the Larson radical of the nest. This substitution broadens the applicability of the result and strengthens its connections with the existing nest algebra literature.

$H$ denotes a (real or complex) Hilbert space of any dimension. $B(H)$ is the space of all bounded, linear operators on $H$. For any set $S$ of projections in $B(H)$, alg $S$ denotes the set of all elements of $B(H)$ which leave invariant the ranges of all elements of $S$. The commutant of $S$ is called the "diagonal" of alg $S$ because it is the intersection of alg $S$ and the set of adjoints of elements in alg $S$. For any $A$ in $B(H), \operatorname{rp}(A)$ denotes the projection on the closure of $R(A)$, the range of $A$. We use $\|\cdot\|$ for the norms in $H$ and $B(H)$.

In all that follows, $\Pi$ will denote a chain of projections in $B(H)$ which contains the elements 0 and $I$. An interval in $\Pi$ is $P_{2}-P_{1}$ where $P_{i} \in \Pi$ for $i=1,2$ and $P_{1}<P_{2}$. A partition of $\Pi$ is a countable set of mutually orthogonal intervals $E_{a}$ of $\Pi$ with $\sum_{a} E_{a}=I$. The Larson radical of alg $\Pi$ is $\{A \in \operatorname{alg} \Pi$ : for every $\varepsilon>0$ there exists a partition $\left\{E_{a}\right\}$ with $\left.\sup _{a}\left\|E_{a} A E_{a}\right\|<\varepsilon\right\}$. We refer the reader to [4 and 5] for further information about the Larson radical, but we need only the definition here. J. R. Ringrose's characterization of the Jacobson radical of alg $\Pi$ for

Received by the editors April 21, 1986 and, in revised form, October 20, 1986.

1980 Mathematics Subject Classification (1985 Revision). Primary 47D25.

Key words and phrases. Nest algebra, CSL algebra, factorization of positive operators. 
a complete chain $\Pi[6]$ motivated Larson's definition and implies that the Jacobson radical is contained in the Larson radical.

REMARK. Elementary arguments show that $\sup _{a}\left\|E_{a} A E_{a}\right\|=\left\|\sum_{a} E_{a} A E_{a}\right\|$ (because of the orthogonality of the intervals), even if $A$ depends on $a$.

THEOREM. Let $\Pi$ be a chain of projections in $B(H)$ with 0 and $I$ in $\Pi$. Let $Q$ be any projection which commutes with every element of $\Pi$, and let $V$ be any element of the Larson radical of alg $\Pi$. Suppose there exists $B$ in $B(H)$ such that $B$ leaves $R(Q)$ invariant, $B+V$ is invertible, $(B+V)^{-1}$ belongs to alg $\Pi$, and $\operatorname{rp}((B+V) Q)$ commutes with $\Pi$. Then $V$ leaves $R(Q)$ invariant. Thus if $V$ is quasinilpotent (in particular, if $V$ is in the Jacobson radical of alg $\Pi), \operatorname{rp}((\lambda I+V) Q)=Q$ for all nonzero scalars $\lambda$ such that $\operatorname{rp}((\lambda I+V) Q)$ commutes with $\Pi$.

Proof. Let $A$ denote $B+V$, and choose $f$ in $R(Q)$. Notice that for all $P$ in $\Pi, P A f$ belongs to $R(\operatorname{rp}(A Q) P)$ because $P$ commutes with $\operatorname{rp}(A Q)$.

Given $\varepsilon>0$, choose a partition of $\Pi$ consisting of intervals $E_{a}$ such that $\sup _{a}\left\|E_{a} V E_{a}\right\|<\varepsilon$. Denote the endpoints of $E_{a}$ as $P_{1}(a)$ and $P_{2}(a)$ with $P_{1}(a)<$ $P_{2}(a)$.

For all $a$, let $h_{a}=A^{-1} P_{2}(a) A f . h_{a}=Q h_{a}$ because $h_{a}$ belongs to

$$
A^{-1}\left(R\left(\operatorname{rp}(A Q) P_{2}(a)\right)\right) \subset A^{-1}(R(A Q)) \subset R(Q) .
$$

Also, $h_{a}=P_{2}(a) h_{a}$ because $A^{-1}$ belongs to alg $\Pi$.

$$
(I-Q) A h_{a}=(I-Q) P_{2}(a) A f=P_{2}(a)(I-Q)(B f+V f)=(I-Q) P_{2}(a) V f .
$$

Also, $(I-Q) A h_{a}=(I-Q) V h_{a}$. Equating these two expressions for $(I-Q) A h_{a}$, we have $(I-Q) V h_{a}=(I-Q) P_{2}(a) V f$, which implies

$$
E_{a}(I-Q) V h_{a}=E_{a}(I-Q) V f \text { for all } a .
$$

For all $a, E_{a}(I-Q) V h_{a}=(I-Q) E_{a} V P_{2}(a) h_{a}=(I-Q) E_{a} V E_{a} P_{2}(a) h_{a}$ (because $E_{a} \leq P_{2}(a)$ and the endpoints of $E_{a}$ are invariant projections for $V$ ) $=(I-Q) E_{a} V E_{a} h_{a}$. Thus, $\sum_{a} E_{a}(I-Q) V h_{a}=\sum_{a}(I-Q) E_{a} V E_{a} h_{a}$. By (*), $\sum_{a} E_{a}(I-Q) V h_{a}=\sum_{a} E_{a}(I-Q) V f=(I-Q) V f$. Combining these last two results,

$$
\begin{aligned}
\|(I-Q) V f\| & =\left\|\sum_{a}(I-Q) E_{a} V E_{a} h_{a}\right\|=\left\|\sum_{a} E_{a}(I-Q) E_{a} V E_{a} A^{-1} P_{2}(a) A f\right\| \\
& \leq\left\|\sum_{a} E_{a}(I-Q) E_{a} V E_{a} A^{-1} P_{2}(a)\right\| \cdot\|A f\| \\
& =\left\|\sum_{a} E_{a}(I-Q) E_{a} V E_{a} A^{-1} E_{a}\right\| \cdot\|A f\| \\
& =\sup _{a}\left\|E_{a}(I-Q) E_{a} V E_{a} A^{-1} E_{a}\right\| \cdot\|A f\|
\end{aligned}
$$

(by the remark preceding the theorem)

$$
\leq \sup _{a}\left\|E_{a} V E_{a}\right\| \cdot\left\|A^{-1}\right\| \cdot\|A f\|<\varepsilon\left\|A^{-1}\right\| \cdot\|A f\| \text {. }
$$


The arbitrariness of $\varepsilon$ now implies that $(I-Q) V f=0$ for all $f$ in $R(Q)$. That is, $R(Q)$ is invariant under $V$.

The final assertion of the theorem follows from the invariance of $R(Q)$ under $\kappa I+V$ and $(\kappa I+V)^{-1}$, the latter having a convergent power series expansion in terms of $V$.

To illustrate the importance of the preceding theorem to the operator factorization problem, we state below a corollary which is easily derived from the preceding theorem using the following observation from [1, p. 405]. Let $S$ be a selfadjoint, positive-definite, bounded, linear operator on $H$. Let $X$ and $Y$ be elements of $B(H)$. The following conditions are equivalent:

(i) There exists $A$ in $B(H)$ such that $A^{*} A=S$ and $\operatorname{rp}(A X)$ commutes with $\operatorname{rp}(A Y)$.

(ii) $\operatorname{rp}(A X)$ commutes with $\operatorname{rp}(A Y)$ for all $A$ such that $A^{*} A=S$.

Let us use the term bordered chain for a chain which contains 0 and $I$.

COROLlaRY 1. Let $T$ be an invertible element of $B(H)$, and let $\Pi$ be any bordered chain of projections. Suppose that $T^{*} T=(\lambda I+V)^{*}(\lambda I+V)$ for some scalar $\lambda$ and element $V$ of the Larson radical of alg $\Pi$ such that $(\lambda I+V)^{-1}$ belongs to alg $\Pi$. Then for any projection $Q$ in the commutant of $\Pi$, if $\operatorname{rp}(T Q)$ commutes with $\Pi$, then $\operatorname{rp}((\lambda I+V) Q) \leq Q$.

Assume from now on that $H$ is separable.

Observe that if the operator $T$ of Corollary 1 is invertible and satisfies $T^{*} T=$ $I+K$ with $K$ in the Macaev ideal, then the well-known factorization theory of Gohberg and Krein [3] may be applied. (In particular, this includes the case that $K$ is a Hilbert-Schmidt operator, which arises in the theory of nonanticipative representations of Gaussian random fields.) If $\Pi$ is a complete, bordered, continuous chain, then a factorization $T^{*} T=(I+V)^{*}(I+V)$ with $V$ a Volterra operator in the Jacobson radical of alg $\Pi$ is provided by the theory of [3]. In this case, $(I+V)^{-1}$ belongs to alg $\Pi$ and the invariance of $R(Q)$ under $V$ is equivalent to $\operatorname{rp}((I+V) Q)=Q$. Thus we have

COROLlaRY 2. Let $S$ be a commutative set of projections in $B(H)$ which contains a complete, continuous, bordered chain $\Pi$. Let $T$ in $B(H)$ be invertible and satisfy $T^{*} T=I+K$ with $K$ in the Macaev ideal. Then the Gohberg-Krein factorization of $T^{*} T$ along $\Pi, T^{*} T=(I+V)^{*}(I+V)$, satisfies $\operatorname{rp}((I+V) P)=P$ for all $P$ in $S$ if and only if $\{\operatorname{rp}(T P): P \in S\}$ is commutative.

Finally, the standard technique of working with the unitary operator $T(I+V)^{-1}$ translates operator factorization results to results about unitary equivalence. (Two sets of projections, $S$ and $\tau$, are unitarily equivalent if there exists a unitary operator $U$ such that $\tau=\left\{U P U^{*}: P\right.$ belongs to $\left.S\right\}$.)

COROllary 3. Let $S$ and $T$ be as in Corollary 2. Then $\{\operatorname{rp}(T P): P$ belongs to $S\}$ is unitarily equivalent to $S$ if and only if it is commutative.

\section{REFERENCES}

1. J. Daughtry and B. Dearden, $A$ test for the existence of Gohberg-Krein representations in terms of multiparameter Wiener processes, J. Funct. Anal. 64 (1985), 403-411.

2. J. Daughtry, Factorization of nonnegative operators along commuting sets of projections, Indiana Univ. Math. J. 35 (1986), 767777. 
3. I. C. Gohberg and M. G. Krein, Theory and applications of Volterra operators in Hilbert space, Amer. Math. Soc., Providence, R. I., 1970.

4. A. Hopenwasser, Hypercausal linear operators, SIAM J. Control and Optim. 6 (1984), 911-919.

5. D. R. Larson, Nest algebras and similarity transformations, Ann. of Math. (2) 121 (1985), 409-427.

6. J. R. Ringrose, On some algebras of operators, Proc. London Math. Soc. (3) 15 (1965), 61-83.

Department of Mathematics, EAst Carolina University, Greenville, North CAROLINA 27858-4353 